\title{
A CONTRIBUIÇÃO DO TRANSPORTE AEROMEDICO NA SOBREVIDA DOS PACIENTES VÍTIMAS DE TRAUMA E AGRAVOS À SAÚDE: UM OLHAR À LUZ DE EVIDÊNCIAS
}

\author{
Gabriel Guembarski FLAVIO'1, Thamyris Lucimar Pastorini GONÇALVES², Bruna \\ Daniella de Sousa de LIMA³, Marcia Eiko KARINO ${ }^{4}$.
}

\section{RESUMO SIMPLES}

O serviço aeromédico através do emprego de aeronaves de asa rotativa ou fixa, vem ganhando cada vez mais destaques nos serviços de saúde, tanto em órgãos públicos quanto privados. Diante do exposto, emergiu-se a seguinte problemática de pesquisa: qual a contribuição do transporte aeromédico na sobrevida de pacientes vítimas de trauma e agravos à saúde? O presente estudo objetivou compreender qual a contribuição do transporte aeromédico na sobrevida de pacientes vítimas de trauma e agravos à saúde. Tratou-se de um estudo de revisão bibliográfica com abordagem descritiva, realizado a partir de artigos publicados nas seguintes plataformas de pesquisa: PubMed, Biblioteca Virtual em Saúde e Literatura LatinoAmericana e do Caribe em Ciências da Saúde. De modo geral, pode-se inferir que o transporte aeromédico contribui para uma melhor assistência à população e consequentemente para o aumento na sobrevida de vítimas de traumas e agravos à saúde. Entretanto, os estudos apontaram que ainda não há conformidade nos resultados elencados, o que dificulta a real dimensão e importância do serviço aeromédico e merecem futuros estudos.

Palavras-chave: Trauma, Atendimento de emergência, Sobrevida, Medicina Aeroespacial.

\section{INTRODUÇÃO}

Atualmente no Brasil, as cidades estão cada vez mais populosas com expansão territorial e surgimento de novos bairros nas periferias das cidades e capitais, dessa forma, também há o aumento significativo de agravos à saúde, no qual exigem um transporte rápido e eficaz até os centros de referência de urgência em saúde (JUNIOR, C. A.G, 2020).

A população vem usando cada vez mais o automóvel para se deslocar a médias e longas distancias, o que acarreta longas filas de congestionamento, horas de espera para se locomoverem poucos quilômetros, afetando negativamente os

\footnotetext{
${ }^{1}$ Enfermeiro residente em urgência e emergência pela Universidade Estadual de Londrina (UEL).

E-mail: gguembarski@hotmail.com

${ }^{2}$ Enfermeira residente em urgência e emergência pela Universidade Estadual de Londrina (UEL).

E-mail: pastorini.thamy@gmail.com

${ }^{3}$ Enfermeira residente em urgência e emergência pela Universidade Estadual de Londrina (UEL).

E-mail: brunadaniella2@hotmail.com

${ }^{4}$ Professora Doutora pela Universidade Estadual de Londrina (UEL).

E-mail: marciak2503@hotmail.com
} 


\section{CONAER \\ CONGRESSO AEROMÉDICO BRASILEIRO}

serviços de urgência que prestam atendimento as pessoas que necessitam de acesso rápido ao serviço de saúde (LEMOS, M.M. 2020).

Em pacientes traumatizados e com agravos clínicos graves a saúde, 0 tempo gasto para o deslocamento da origem até a unidade de referência é crucial para estabelecer um tratamento eficaz e definitivo no menor intervalo de tempo possível, garantindo assim a maior sobrevida do indivíduo (LEMOS, M.M. 2020).

O serviço de transporte aeromédico está inserido no sistema de atendimento médico pré-hospitalar de urgência e emergência, sendo regulamentado pelas portarias do Ministério da Saúde GM/MS 2.048 de 05 de novembro de 2002 e 1.863/GM, de 29 de julho de 2003 (GOMES, et al., 2013).

Desde então, o serviço aeromédico através do emprego de aeronaves de asa rotativa ou fixa, vem ganhando cada vez mais destaque nos serviços de saúde, tanto em órgãos públicos quanto privados, contribuindo para uma melhor e mais rápida assistência ao paciente grave.

Diante do exposto, emergiu-se a seguinte problemática de pesquisa: qual a contribuição do transporte aeromédico na sobrevida de pacientes vítimas de trauma e agravos à saúde?

O presente estudo objetivou compreender qual contribuição do transporte aeromédico na sobrevida de pacientes vítimas de trauma e agravos à saúde.

\section{METODOLOGIA}

Tratou-se de um estudo de revisão bibliográfica com abordagem descritiva, realizado a partir de artigos publicados nas seguintes plataformas de pesquisa: PubMed, Biblioteca Virtual em Saúde (BVS) e Literatura Latino-Americana e do Caribe em Ciências da Saúde (LILACS). Ambas são bases de dados utilizadas para suporte nas pesquisas avançadas, as quais promovem o acesso a informação cientifica, especializada e técnica em saúde.

A estratégia metodológica utilizada foram artigos com os critérios de inclusão: artigos publicados nos últimos dez anos, disponíveis na língua portuguesa e inglesa, artigos de revisão sistemática e pesquisa de campo que estavam disponíveis em texto completo de forma paga ou gratuita. Como critérios de exclusão, foram eliminados os artigos que não correspondiam aos objetivos da temática e que apresentavam textos incompletos. Ressalta-se que foram utilizados os Descritores em ciência da saúde (DeCS): emergência, helicóptero e transporte aeromédico, bem 
como o Medical Subject Headings (MeSH): medical, emergency, helicopter e aeromedical transport. Utilizou-se também o operador booleano AND ou OR.

Durante a busca, na base de dados PubMed foram encontrados no total 32 artigos, sendo selecionados três deles, enquanto na BVS foram encontrados sete artigos, e que apenas dois se encaixaram nos critérios de inclusão, e no LILACS foram encontrados 25 artigos, e filtrado apenas três, totalizando assim 8 artigos aptos para a discussão.

\section{RESULTADOS E DISCUSSÃO}

A utilização do serviço aeromédico é um meio de assistência à saúde que promove suporte avançado de vida, capaz de oferecer atendimento rápido e eficaz à população, garantindo a pessoa assistida, um menor tempo de deslocamento ao serviço de saúde especializado e assim, dar continuidade ao seu tratamento definitivo (NASCIMENTO et al., 2018).

Partindo desse pressuposto, destaca-se o estudo de Nascimento et al., (2020) que diz respeito ao serviço aeromédico (SAM) a população idosa, nele pôde-se observar que a porcentagem de óbitos foi relativamente baixa $(21,9 \%)$ em relação ao total de atendimentos, de 214 indivíduos. Dessa maneira, destacou-se a importância de tal serviço a essa população, pois além de oferecer um translado seguro e rápido a instituição de saúde, ofertou também um atendimento imediato de urgência.

Em contrapartida, em uma pesquisa realizada em Campinas no ano de 2014, em análise de prontuários e fichas médicas de pacientes atendidos pelo SAM, verificou que o índice de mortalidade no atendimento aéreo não apresentou redução quando comparado ao serviço terrestre. Nesse sentido, a efetividade do serviço ocorreu apenas em distâncias relativamente grandes, onde o acionamento da equipe de saúde terrestre foi considerado inábil (CARDOSO et al., 2014).

Assim, de acordo com Cardoso et al., (2014) questiona-se, se o tempo resposta do atendimento, desde o chamado até a chegada da equipe do SAM no local, está relacionado ou não com a gravidade do quadro clínico do paciente, uma vez que, tal fator está diretamente ligado ao desfecho clínico do caso. Dessa maneira, pode dizer que o uso desse serviço não garante uma sobrevida a pessoa atendida. 
Em compensação, foi possível identificar também que, o menor tempo de transporte entre o local da ocorrência ao serviço de saúde, associado a intervenções necessárias em cena, garante ao paciente um menor tempo de internação, além de reduzir a mortalidade e garantir um melhor potencial de sobrevida para ele (GALVAGNO et al., 2015).

Destaca-se também, o uso do serviço aeromédico para transporte de agravos clínicos, como emergência cardiológica e acidente vascular cerebral. Em um estudo foi notado um custo-benefício favorável em transportes aeromédicos de pacientes com AVC isquêmico em tempo de trombólise (SHACHAR; SABAN MOR, 2020).

Além disso, enumerou-se as principais causas do acionamento do SAM, que foram destinados ao atendimento a traumas, entre eles: motocicletas, colisões automobilísticas, queda de plano elevado e atropelamentos. Isso porque, no atendimento ao traumatizado, espera-se encontrar lesões graves e ameaçadoras a vida, o que torna essencial a presença do médico em cena, sendo assim, justificado o emprego da aeronave, já que em algumas configurações de serviços préhospitalares terrestres não está incluso o acompanhamento deste profissional. Desta forma, ficou evidente um aumento na sobrevida dos pacientes atendidos pelo aeromédico quando comparado ao serviço terrestre (Cardoso et al., 2014).

Segundo Risgaard et al., (2020), o paciente traumatizado que utilizou do serviço aeromédico, obteve em seu período de internação hospitalar uma redução de mortalidade entre $11 \%$ e $17 \%$, bem como apresentou uma melhora significativa da sobrevida em relação ao paciente atendido pelo serviço terrestre.

\section{CONSIDERAÇÕES FINAIS}

Nesta análise, pode-se inferir que o transporte aeromédico contribui para uma melhor assistência à população e consequentemente, para o aumento da sobrevida de vítimas de traumas e agravos à saúde, ofertando um tempo de atendimento mais rápido e ágil, principalmente à longas distâncias. Entretanto, os estudos apontaram que ainda não há uma conformidade nos resultados elencados, o que dificulta a real dimensão e importância do serviço aeromédico.

Assim, conclui-se que ainda são necessários novos estudos que abordem tal temática, bem como, pesquisas a respeito da sobrevida desses pacientes. Ressaltase que este estudo poderá servir de subsídio e embasamento para novas pesquisas da temática em questão. 


\section{CONAER \\ CONGRESSO AEROMÉDICO BRASILEIRO}

\section{REFERÊNCIAS}

1. CARDOSO, R. G; FRANCISCHINI, C. F; RIBERA, J. M; VANZETTO, R; FRAGA, G. P. Resgate aeromédico a traumatizados: experiência na região metropolitana de Campinas, Brasil: Rev. Col. Bras. Cir., v. 41, n. 4, p. 236-244, 2014. Disponível em: https://pesquisa.bvsalud.org/portal/resource/pt/lil-724110. Acesso em: 25 de jul. 2021.

2. GOMES, M. A. V; ALBERTI, L. R; FERREIRA, F. L; GOMES, V. M. Aspectos históricos do transporte aeromédico e da medicina aeroespacial - revisão. Rev. Med. Minas Gerais, v. 23, n. 1, p. 116-123, 2013. Disponível em: http://www.rmmg.org/exportar-pdf/20/v23n1a18.pdf. Acesso em: 27 de ago. 2021.

3. JUNIOR, C. A. G. História do Resgate Aeromédico no Brasil. In SUEOKA, J. S; FREIXO, J. A. A; TAVERNA, M. (org.). Transporte e Resgate Aeromédico. $1^{\text {a }}$ ed. Rio de Janeiro: Guanabara Koogan, 2020. 1. p. Cap.1. Acesso em: 23 jul. 2021.

4. JUNIOR, S. M. G, et al. Helicopter emergency medical services for adults with major trauma. Rev. Cochrane Database Syst, v. 28, n. 3. 2013. Disponível em: https://pubmed.ncbi.nlm.nih.gov/26671262/. Acesso em: 25 de jul. 2021.

5. LEMOS, M.M. Resgate Aeromédico. In SUEOKA, J. S; FREIXO, J. A. A; TAVERNA, M. (org.). Transporte e Resgate Aeromédico. 1. ed. Rio de Janeiro: Guanabara Koogan, 2020. 90 p. Cap. 14. Acesso em: 23 de jul. 2021.

6. NASCIMENTO, K. C. et al. Idosos atendidos por um serviço aeromédico. Rev. Bras. Geriatr. Gerontol, v. 21, n. 1, p. 82-90, 2018. Disponível em: https://pesquisa.bvsalud.org/portal//resource/pt/biblio-898819. Acesso em: 25 de jul.2021.

7. RISGAARD, B; DRAEGERT, C; BAEKGAARD, J. S; STEINMETZ, J; RASMUSSEN, J. Impact of Physician-staffed helicopter on Pre-hospital Patient Outcomes: A systematic review; Rev. Acta Anesthesiol Scand, v. 64, n. 5, p. 691704. 2020. Disponível em: https://pubmed.ncbi.nlm.nih.gov/31950487/. Acesso em: 25 de jul.2021.

8. TAL, S; MOR, S. The impact of helicopter emergency medical servisse on acute ischemic stroke patients: A systematic review. Am J Emerg Med, V. 42, p. 178-187. 2021. Disponível em: https://pubmed.ncbi.nlm.nih.gov/32089368/. Acesso em: 25 de jul. 2021. 